\title{
A Layered Architecture for Vehicular Delay-Tolerant Networks
}

\author{
Vasco N. G. J. Soares ${ }^{1,2,3}$, Farid Farahmand ${ }^{4}$, and Joel J. P. C. Rodrigues ${ }^{1,2}$, \\ ${ }^{1}$ Instituto de Telecomunicações, Next Generation Networks and Applications Group, Portugal \\ ${ }^{2}$ Department of Informatics, University of Beira Interior, Covilhã, Portugal \\ ${ }^{3}$ Superior School of Technology, Polytechnic Institute of Castelo Branco, \\ Castelo Branco, Portugal \\ ${ }^{4}$ Department of Engineering Science, Sonoma State University, CA, USA \\ vasco_g_soares@est.ipcb.pt; farid.farahmand@sonoma.edu; joeljr@ieee.org
}

\begin{abstract}
Vehicular Delay-Tolerant Network (VDTN) is a new network architecture based on the concept of Delay Tolerant Networks (DTN). It aims to be an architecture that handles non-real time applications at low cost, under unreliable conditions, enabling connectivity in diverse scenarios, using vehicles to carry data between terminal nodes. For example, it can be applied in rural and remote areas, or in emergency scenarios. This paper proposes a layered architecture for VDTNs, using out-of-band signaling, based on the separation of the control plane and data plane. It presents the layers interactions and the envisioned protocols required at each layer. The paper provides a deep understanding of the characteristics of VDTN and reveal some design issues in its modeling, leading to insights for future theoretic study and protocol design.
\end{abstract}

\section{Introduction}

Delay Tolerant Networks (DTNs) are a class of networks that enable communication where connectivity issues like sparse connectivity, intermittent connectivity, long or variable delay, asymmetric data rate, high latency, high error rates, and even no end-to-end connectivity exist [1]. The use of Internet reliable transport and routing protocols on such scenarios may not be appropriate [2].

The DTN architecture implements a store-andforward paradigm by overlaying a protocol layer, called bundle layer, that it is meant to provide internetworking on heterogeneous networks operating on different transmission media [3]. At the edge of each remote area network, a border system has an application layer gateway to terminate applications and produce data bundles. Bundles are relayed hop by hop between gateways through a series of fixed and mobile nodes. Intermediate nodes are responsible for storing bundles and carrying them while a contact is not available.

DTNs have been applied to performance-challenged scenarios characterized by the lack of guaranteed connectivity and the typically low frequency of encounters between a given pair of nodes within the network (as may be seen in Figure 1). Examples of these proposals are interplanetary networking [2, 4], data MULEs [5, 6], underwater networks [7], and wildlife tracking sensor networks like ZebraNet [8].

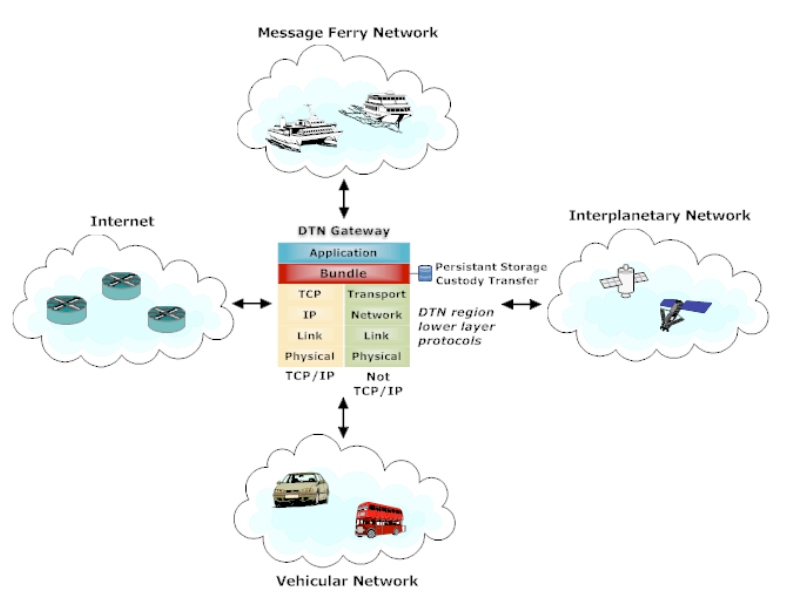

Figura 1. DTN bundle concept and DTN gateway layer interactions.

The DTN architecture concept was also extended to transit networks, called Vehicular DTN (VDTN). In these networks vehicles (e.g., cars, buses, and boats) are exploited to offer a message relaying service by moving around the network and collecting messages from source nodes. A number of projects have been based on this general concept. For example, the 
Message Ferry project to develop a data delivery system in disconnected areas $[9,10]$. Another example is the DakNet project proposed to provide low-cost connectivity to the Internet to rural villages in India [11]. Vehicular Networks have also been used for traffic condition notifications, accident warnings, automatic tolling, free parking spots information, advertisements, and for example to gather data collected by vehicles like road pavement defects [12$15]$.

In this paper we exemplify the use of a VDTN to provide asynchronous Internet access on a rural and remote regions scenario. The VDTN architecture model is based on the following three node types mentioned in Figure 2: terminal nodes, mobile nodes, and relay nodes. Terminal nodes are access points to the VDTN and may be located in isolated regions. They provide the connection to end-users, allowing them to use non-real time applications. At least, one of the terminal nodes may have a direct access to the Internet. Mobile nodes (e.g., vehicles) are responsible for physically carrying data between terminal nodes. Relay nodes are fixed devices located at crossroads, with low-power requirements and store-and-forward capabilities. They allow mobile nodes that pass by to collect and leave data on them. Mobile nodes can also exchange information with one another.

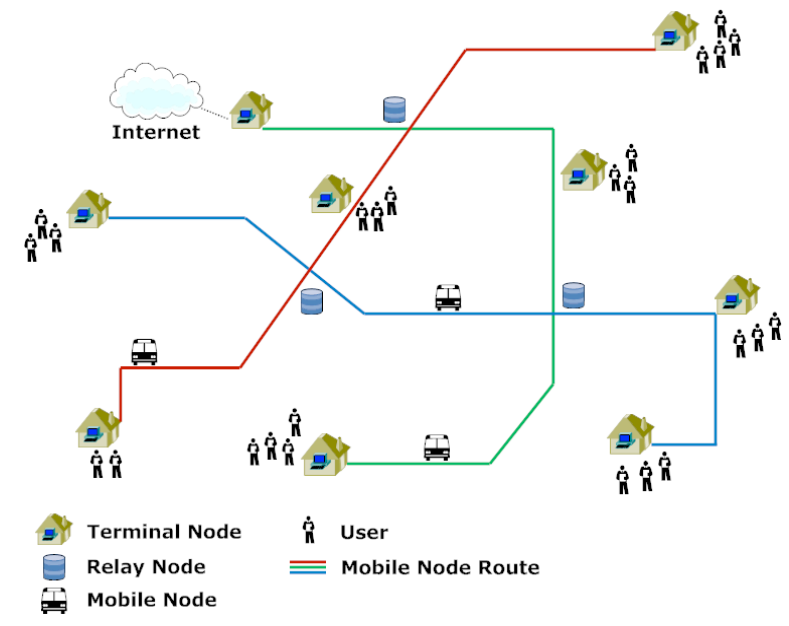

Figure 2. Example of a Vehicular DelayTolerant Network providing connection on rural and remote regions.

VDTN is a very challenging topic that introduces specific network issues and problems, such as the following: architecture (naming and addressing), node design (power, storage capacity, range, speed, physical link), node type (mobile, stationary), node interactions, node cooperation, network topology (known or not), mobility pattern (deterministic, stochastic, predictable, etc.), scheduling, traffic (static, dynamic), routing protocols, bundle format, caching mechanisms, security, and supported applications.

The key contribution of this paper is the proposal of a VDTN layered architecture with data plane and control plane separation. This approach suggests the use of control information at the connection setup phase. It will adjust a connection's characteristics to support appropriate transmission and reception of application data, improving the overall network performance. A well-defined model with well-defined interfaces between the layers can lead to insight for future theoretic study, protocol design, implementation techniques, specifications and functionalities of a VTDN.

The reminder of this paper is organized as follows. Section 2 discusses the layered architecture of IP-overVDTN. Section 3 describes each layer of the VDTN layered architecture, separating them into a data plane and a control plane. Finally, Section 4 concludes the paper.

\section{IP-over-VDTN Layered Architecture}

The Vehicular Delay-Tolerant Network layered architecture follows the Open Systems Interconnection (OSI) reference model, and the Transmission Control Protocol/Internet Protocol (TCP/IP) architecture. As may be seen in Figure 3, it shows the VDTN proposed layered architecture, in comparison with OSI model, TCP/IP and DTN architectures. VDTN protocol stack implements the two lower layers of OSI model, since the VDTN layer supports physical layer services and functions, and connection management services related to the data link layer.

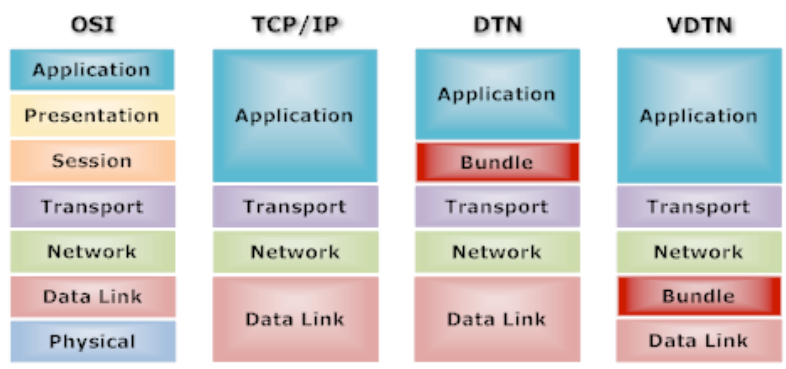

\section{Figure 3. Generic network architecture layers: comparison of OSI, TCP/IP, DTN, and VDTN} models.

In our opinion, the intermittent connectivity and the discrete nature of transfer opportunities in the transportation system of the VDTN application 
scenario, motivates the routing of large size messages instead of small size IP packets. This results in fewer packets processing and routing decisions, which can be translated to less complexity, lower cost and energy savings.

VDTN also defines the bundle as its protocol data unit. However, the bundle layer is called Bundle Aggregation and De-aggregation (BAD) Layer (as shown in Figure 4). It is located below the network layer in order to aggregate incoming IP data packets into bundle messages. Even though, we assume that messages are based on the IP paradigm and the connected access network to the VDTN is an IP network, this architecture is general enough to support any other network layer protocol. VDTN supports any network protocol since bundles may be created at edge of the network.

Since VDTN technology is comprised only in the two lower layers of the OSI model, this also leads to a simpler and faster processing of protocol data units. BAD layer allows mobile nodes to upload and download bundles to/from other mobile nodes, relay nodes and terminal nodes.

Figure 4 presents the proposed VDTN layered architecture. In this illustration we separate the data plane functionalities and protocols from those of the control plane. The data plane assembles and processes the data bundles (DBs), while the control plane is responsible for the transmission of control bundles (CBs) out-of-band.

The use of distinct planes suggests that they can operate independently using their own layers and protocols. Therefore, we can envision DBs and CBs being encoded and transmitted using different transmission technologies.

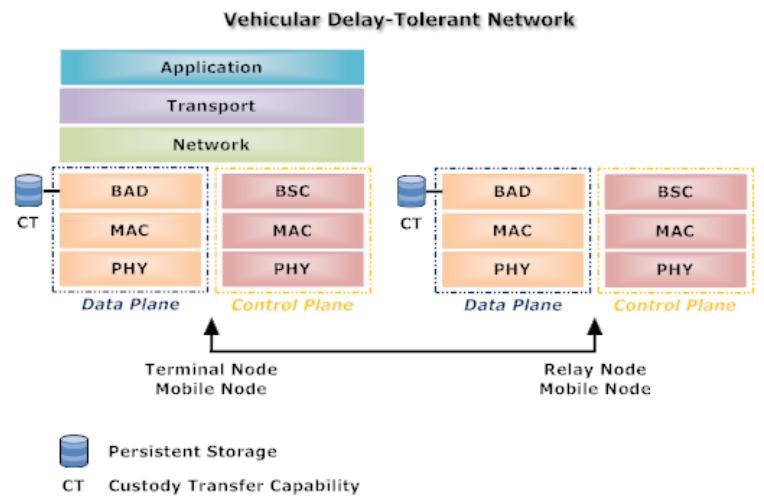

Figure 4. IP-over-VDTN layered architecture.

The CBs are used in an orderly and meaningful way to exchange control information between terminal nodes, relay nodes and mobile nodes. They are applied on the connection setup phase in order to determine and adjust a connection's requested characteristics (e.g., node type, speed, physical link, range, power, storage capacity, bundle format and size, delivery options, security requirements). After the successful exchange of control information, the DBs can be transmitted between the nodes.

Terminal nodes implement all the layers of the VDTN protocol stack and are able to do heavy data processing, so they can implement more complex functions and services. On the contrary, due to possible buffer capacity (memory), and energy constraints of relay nodes and mobile nodes, they implement only the lower layers, offering simple functions. In this manner, bundles will be quickly processed and transmitted over the network. Network intelligence will be concentrated at the edge of the network.

It is also possible that a mobile node may implement the full protocol stack. This enables a scenario where, for instance, it would be possible to do a web search inside a vehicle [16] (e.g., a bus) complementing the above described VDTN architecture model.

\section{VDTN Layered Architecture}

Next subsections present the basic functionalities of each layer in the data and control planes. We describe the layered architecture of each plane in a consistent order with the packet flow, starting with the data plane (Figure 5).

\subsection{Data Plane}

The data plane is responsible for the transport of incoming packets, which are aggregated into data bundles, from a source node to a single or multiple destination nodes.

3.1.1. Bundle Aggregation and De-aggregation (BAD) Layer. Following DTNs architecture, VDTN also implements a store-and-forward message (bundle) switching based on persistent storage.

At the source terminal node, the BAD layer aggregates incoming IP packets with the same properties (e.g., destination terminal node IP address) into DBs, and at the destination terminal node this layer does the inverse process, de-aggregating received DBs into individual IP packets (Figure 5). When an IP packet arrives, it is decoded, its properties are evaluated, and its destination address is translated to a VDTN terminal node address. IP packets with similar properties are aggregated together to form the data bundle payload.

There are several possibilities for DB assembly 
techniques. For example, in application-based bundle assembly, a DB is created by aggregation of IP packets that transport data for the same destination application. Another criteria for bundle assembly can be quality of service (QoS), which can be used to implement a VDTN QoS scheme for traffic prioritization. Under this technique, combining IP packets of different classes into the same bundle creates a DB. IP packets are placed in a decreasing order according to their class from the head to the tail's bundle. This technique has some similarities with the "priority classes" scheme defined for the DTN architecture [3]. All the above techniques take into account a threshold that establishes a limit to the maximum number of IP packets contained in each bundle (the bundle size).

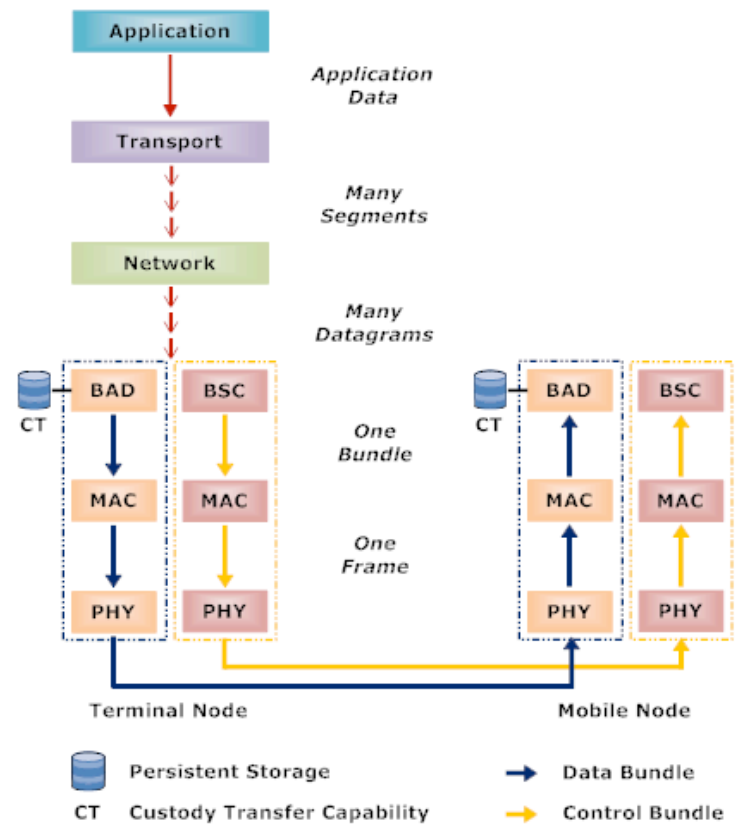

Figure 5. Example of the transmission of control bundles and data bundles, between a terminal node and a mobile node.

DTN custody transfer [3] is also the proposed reliability scheme for VDTN, and it is implemented at the BAD layer. Custody transfer uses hop-by-hop reliability to enhance end-to-end reliability. However, it does not provide guaranteed end-to-end reliability. This can only be accomplished if a source node requests both custody transfer and return receipt. The DTN architecture supports several more delivery options [3], that may also be available on VDTN. Furthermore, applications may select delivery options, and at the BAD layer, a data bundle can be created by the aggregation of IP packets with the same delivery options. Then, using this criterion, another technique for a DB assembly may be proposed.

To prevent the carriage of unauthorized traffic, it is necessary to authenticate the terminal nodes, relay nodes, and mobile nodes. DBs and CBs integrity can also be protected. To obtain these security requirements, we can rely on the link technologies native security methods, or implement at this layer the mechanisms proposed at the security component of DTN architecture [3]. Security deployment increases processing costs, and this will have a negative impact on energy savings.

If different link technologies, with its correspondent Maximum Transmission Units (MTU), can be used for DB transmission, it is possible to fragment a DB while it is in transit. Fragmentation can also occur when a bundle is only partially transmitted. Therefore, the BAD layer at the final destination (terminal node) is responsible for reassembling the fragments, as well as executing re-sequencing (sequencing verification). The default size for a data bundle is based on the MTU of the link technology used.

Due to the medium transmission error rate in this DTN like scenario, BAD layer also provides the means to detect and correct corrupt data on incoming data bundles. For example, if flooding based routing is used, it can cause a situation where more than one copy of a particular DB can arrive at the destination node. Even if all of these copies are corrupted, their combined information can be used to correct or minimize errors, obtaining as much error free information as possible. Power constraints make this function only available at terminal nodes and eventually mobile nodes.

3.1.2. Media Access Control (MAC) Layer. The MAC layer provides functional and procedural means to transfer data between our VDTN network entities in point-to-point and point-to-multipoint communications. In addition, for framing and media access control, this layer contains flow control mechanisms that are used between terminal nodes, relay nodes and mobile nodes, to pace the rate at which DBs are sent. Though MAC layer may provide the possibility to detect and eventually correct errors that may occur in the physical layer, these functions are not desirable at this layer, so they are only performed at the BAD layer.

The link technologies that are used to support the DBs exchanged between terminal nodes, mobile nodes, and relay nodes, operates the normal functionalities defined for the two lower layers of the OSI reference model. Thus, each technology has its specific MAC and PHY layers. Examples of link technologies include, but are not limited to: IEEE 802.11, Dedicated Short Range Communications (DSRC), Ultra- 
wideband (UWB), and UMTS. We can also consider a scenario where VDTN nodes have more than one of these technologies available, and exchanging control information (CBs), it is decided what link technology will be employ at a particular contact established between two nodes.

Technology or technologies available on terminal nodes, mobile nodes, and relay nodes will be chosen, based on the following criteria: node type (mobile, stationary), node design (power, storage capacity, speed), link characteristics (bandwidth, range, error rate, power consumption), and economical considerations.

3.1.3. Physical (PHY) Layer. The physical layer is responsible for the actual transport of DBs from one node to another. Even though physical layer functions are essential for the overall performance of the link technology, since they are part of the implementation of a specific link technology, it is not relevant to discuss them in the context of this work.

\subsection{Control Plane}

As above-mentioned, the separation between data and control planes in Vehicular Delay-Tolerant Networks was inspired by the possibility of mobile, relay and terminal nodes exchange control information at the connection setup phase. Then, in order to determine and adjust a connection requested characteristics to support appropriate transmission and reception of DBs, splitting control and data planes will improve the overall performance of the VDTN.

3.2.1. Bundle Signaling Control (BSC) Layer. The Bundle Signaling Control (BSC) layer provides a signaling protocol for use at the connection setup phase. The protocol allows two nodes to exchange control information (using CBs), to discover each other's characteristics. For example, they can exchange information related with node type, speed, power and storage capacity constraints, and supported link technologies. Based on this information, nodes make an agreement settling, namely, the link technology that will be used to carry the DBs, if the communication will be point-to-point or point-to-multipoint, the bundle size, and the storage space reserved for these DBs.

It is also possible to use control information to specify the traffic prioritization, delivery options, and security requirements. This layer also includes routing algorithms [17, 18] used to decide the path for the incoming data bundles. The BSC layer has an essential role on the overall network performance.
3.2.2. Media Access Control (MAC) and Physical (PHY) Layers. In general we assume that control bundles have a small size, require low bandwidth, and are less prone to transmission errors. Consequently, in a scenario where a VDTN node has support for different link technologies, CBs can be carried out-ofband using a specific predetermined low-powered, low bandwidth technology, to set up a data plane connection. Based on these control messages exchange, the most adequate transmission technology will be chosen to transmit the corresponding DBs. This leads to important energy saving gains that are essential to relay nodes, and improves the overall network performance.

The above-mentioned information about link technologies for the data plane, and their MAC and PHY layer functionalities and procedures, remains valid here. So, these layers are not further discussed.

\section{Conclusions}

This paper proposed and presented a deep description of a layered architecture for Vehicular Delay-Tolerant Networks. The motivation for this approach comes from the complexity of the VDTN system architecture and their characteristics. Based on it, a separation of data plane and control plane, using out-of-band signaling was considered. In addition, insights on the layers protocols and services were provided.

This layered model can be helpful to understand the interactions among VDTN nodes, and be used to settle protocol requirements guiding their future design and development.

Based on this new VDTN architecture, new challenges may be addressed. Then, the following topics may be considered for future work: bundle assembly algorithms, considering quality of service or not, signaling protocols, and error control and reliability.

\section{Acknowledgements}

Part of this work has been supported by the Instituto de Telecomunicações, Next Generation Networks and Applications Group, Covilhã Delegation, Portugal, in the framework of the Project VDTN@Lab, and by the Euro-NF Network of Excellence of Seven Framework Programme of EU. 


\section{References}

[1] K. Fall, "A Delay-Tolerant Network Architecture for Challenged Internets," in ACM SIGCOMM, Karlsruhe, Germany, August 25 - 29, 2003.

[2] S. Burleigh, A. Hooke, L. Torgerson, K. Fall, V. Cerf, B. Durst, K. Scott, and H. Weiss, "Delay-Tolerant Networking: An Approach to Interplanetary Internet," in IEEE Communications Magazine, vol. 41, 2003, pp. 128-136.

[3] V. Cerf, S. Burleigh, A. Hooke, L. Torgerson, R. Durst, K. Scott, K. Fall, and H. Weiss, "Delay-Tolerant Networking Architecture," RFC 4838, April 2007, [Online]. Available: ftp://ftp.rfc-editor.org/innotes/rfc4838.txt.

[4] I. F. Akyildiz, Ö. B. Akan, C. Chen, J. Fang, and W. Su, "Interplanetary Internet: State-of-the-Art and Research Challenges," Computer Networks, vol. 43, pp. 75-112, October 2003

[5] R. C. Shah, S. Roy, S. Jain, and W. Brunette, "Data MULEs: Modeling a Three-Tier Architecture for Sparse Sensor Networks," in First IEEE International Workshop on Sensor Network Protocols and Applications (SNPA 2003), Anchorage, AK, USA, May 11, 2003, pp. 30-41.

[6] S. Jain, R. Shah, W. Brunette, G. Borriello, and S. Roy, "Exploiting Mobility for Energy Efficient Data Collection in Wireless Sensor Networks," ACM/Kluwer Mobile Networks and Applications (MONET), vol. 11, pp. 327-339, June 2006

[7] J. Partan, J. Kurose, and B. N. Levine, "A Survey of Practical Issues in Underwater Networks," in 1st ACM International Workshop on Underwater Networks, in conjunction with ACM MobiCom 2006, Los Angeles, California, USA, Sep. 25, 2006, pp. 17 - 24.

[8] P. Juang, H. Oki, Y. Wang, M. Martonosi, L. S. Peh, and D. Rubenstein, "Energy-Efficient Computing for Wildlife Tracking: Design Tradeoffs and Early Experiences with ZebraNet," ACM SIGOPS Operating Systems Review, vol. 36, pp. 96 - 107, 2002.

[9] W. Zhao, M. Ammar, and E. Zegura, "A Message Ferrying Approach for Data Delivery in Sparse Mobile Ad Hoc Networks," in The Fifth ACM International Symposium on Mobile Ad Hoc Networking and Computing (MobiHoc 2004), Roppongi Hills, Tokyo, Japan, May 24-26, 2004, pp. 187-198.
[10] W. Zhao and M. H. Ammar, "Message Ferrying: Proactive Routing in Highly-Partitioned Wireless Ad Hoc Networks," in The Ninth IEEE Workshop on Future Trends of Distributed Computing Systems, San Juan, Puerto Rico, May 28-30, 2003, pp. 308-314.

[11] A. Pentland, R. Fletcher, and A. Hasson, "DakNet: Rethinking Connectivity in Developing Nations," in IEEE Computer, vol. 37, 2004, pp. 78-83.

[12] R. Tatchikou, S. Biswas, and F. Dion, "Cooperative Vehicle Collision Avoidance using Inter-vehicle Packet Forwarding," in IEEE Global Telecommunications Conference (IEEE GLOBECOM 2005), St. Louis, MO, USA, 28 Nov. - 2 Dec., 2005.

[13] I. Leontiadis and C. Mascolo, "GeOpps: Geographical Opportunistic Routing for Vehicular Networks," in IEEE International Symposium on a World of Wireless, Mobile and Multimedia Networks 2007 (WoWMoM 2007), Espoo, Finland, 18-21 June, 2007, pp. 1-6.

[14] O. Brickley, C. Shen, M. Klepal, A. Tabatabaei, and D. Pesch, "A Data Dissemination Strategy for Cooperative Vehicular Systems," in Vehicular Technology Conference 2007 (VTC2007), Dublin, 2007, pp. 25012505 .

[15] L. Franck and F. Gil-Castineira, "Using Delay Tolerant Networks for Car2Car Communications," in IEEE International Symposium on Industrial Electronics 2007 (ISIE 2007), Vigo, Spain, 4-7 June, 2007, pp. 25732578 .

[16] A. Balasubramanian, Y. Zhou, W. B. Croft, B. Levine, and A. Venkataramani, "Web Search From a Bus," in ACM International Conference on Mobile Computing and Networking (ACM MobiCom) - Proceedings of the Second Workshop on Challenged Networks (CHANTS 2007), Montreal, Quebec, Canada, September 9-14, 2007, pp. 59 - 66 .

[17] A. Boukerche, Handbook of Algorithms for Wireless Networking and Mobile Computing. Chapman \& Hall/CRC Computer and Information Science Series, 2005.

[18] Z. Zhang, "Routing in Intermittently Connected Mobile Ad Hoc Networks and Delay Tolerant Networks: Overview and Challenges," IEEE Communications Surveys \& Tutorials, vol. 8, pp. 24-37, 2006. 